\title{
Brain structure and function predict adherence to an exercise intervention in older adults
}

Timothy P. Morris ${ }^{a}$, Agnieszka Burzynska ${ }^{b}$, Michelle Voss ${ }^{c}$, Jason Fanning ${ }^{d}$, Elizabeth A. Salerno ${ }^{\mathrm{e}}$, Ruchika Prakash ${ }^{f}$, Neha P. Gothe ${ }^{\mathrm{g}, \mathrm{h}}$, Susan Whitfield-Gabrielia,i, Charles H. Hillman ${ }^{\mathrm{a}, \mathrm{j}}$, Edward McAuley, $\mathrm{h}$, Arthur F. Kramer ${ }^{\mathrm{a}, \mathrm{k}}$

a Department of Psychology, Northeastern University, Boston, MA, United States

${ }^{b}$ Department of Human Development and Family Studies, Colorado State University, USA

${ }^{c}$ Dept of Psychology, University of lowa

d Department of Health and Exercise Science, Wake Forest University, North Carolina, USA

e Division of Public Health Sciences, Department of Surgery, Washington University School of Medicine in St. Louis, St. Louis, MO

${ }^{f}$ Department of Psychology, Ohio State University

${ }^{9}$ Beckman Institute for Advanced Science and Technology, University of Illinois at

Urbana-Champaign, United States

h Department of Kinesiology and Community Health, University of Illinois at UrbanaChampaign, Urbana, IL, United States

'McGovern Institute for Brain Research, Department of Brain and Cognitive Sciences, Massachusetts Institute of Technology, Cambridge, MA, United States.

jDepartment of Physical Therapy, Movement, and Rehabilitation Sciences, Northeastern University, Boston, MA, United States

${ }^{\mathrm{k}}$ Beckman Institute for Advanced Science and Technology, University of Illinois at Urbana-Champaign, United States

Corresponding author: Dr. Timothy P. Morris, Department of Psychology, Northeastern University, 606 ISEC, 360 Huntington Avenue, Boston, 02115, United States.

t.morris@northeastern.edu

\begin{abstract}
Individualized and precision medicine approaches to exercise for cognitive and brain health in aging have the potential to improve intervention efficacy. Predicting adherence to an exercise intervention in older adults prior to its commencement will allow for adaptive and optimized approaches that could save time (no need to demonstrate failure before changing course) and money (cost of de-implementing approaches that do not work for certain individuals) which ultimately could improve health outcomes (e.g., preventative medicine approaches prior to the onset of symptoms). Individual differences in brain structure and function in older adults are potential proxies of brain and brain reserve or maintenance and may provide strong predictions of adherence. We hypothesized that brain-based measures would predict adherence to a six-month randomized controlled trial of exercise in older adults, alone and in combination with psychosocial, cognitive and health measures. In 131 older adults (aged $65.79 \pm 4.65$ years, $63 \%$ female) we found, using regularized elastic net regression within a nested
\end{abstract}


cross-validation framework, that brain structure (cortical thickness and cortical surface area) in somatosensory, inferior temporal, and inferior frontal regions and functional connectivity (degree count) in primary information processing (somatosensory, visual), executive control, default, and attentional networks, predicted exercise adherence $\left(R^{2}=\right.$ $0.15, p<0.001)$. Traditional survey and clinical measures such as gait and walking selfefficacy, biological sex and perceived stress also predicted adherence $\left(\mathrm{R}^{2}=0.06, p=\right.$ $0.001)$ but a combined multimodal model achieved the highest predictive strength $\left(\mathrm{R}^{2}=\right.$ $0.22, p<0.001)$. Neuroimaging features alone can predict adherence to a structured group-based exercise intervention in older adults which suggests there is substantial utility of these measures for future research into precision medicine approaches. The best performing model contained multimodal features suggesting that each modality provided independent relevant information in the prediction of exercise adherence.

\section{Introduction}

Physical activity and structured exercise have received a lot of attention as potential efficacious interventions to improve or maintain brain health with advancing age (Gomes-Osman et al., 2018; Kelly et al., 2014; Kramer et al., 2003). Despite millions of government and private dollars being spent on understanding how physical activity can improve or maintain brain function across the lifespan, over a third of the US population do not engage in sufficient physical activity (Du et al., 2019; Guthold et al., 2018; Kohl et al., 2012), a statistic which continues to increase (Du et al., 2019; Guthold et al., 2018). This physical inactivity pandemic (Andersen et al., 2016; Kohl et al., 2012; Pratt et al., 2020 ) is estimated to cost private and public health-care systems $\$ 53.8$ billion per year and a further $\$ 13.7$ billion in productivity losses due to physical inactivity-related deaths each year (Ding et al., 2016, 2017).

Previous research on understanding the adoption and maintenance of physical activity has leveraged psychological and psychosocial theories (Brand \& Cheval, 2019). In experimental studies, several psychosocial, behavioral and demographic measures were shown to correlate with exercise adherence, such as self-efficacy (Garcia \& King, 1991; McAuley et al., 2011), self-regulation (De Bruin et al., 2012; Dishman et al., 2014), social support, perceived benefits and biological sex (males adhered more than females) (Cadmus-Bertram et al., 2014), higher baseline physical activity outside of the intervention (Arkkukangas et al., 2018) as well as depression, fatigue and general perceived health (Flegal et al., 2007). Additionally, greater cognitive resources, particularly executive functions, known to depend on cortical structural integrity (Burzynska et al., 2011), have been associated with engagement in exercise (Cheval, Daou, et al., 2020; Cheval, Orsholits, et al., 2020). Based on these results, a multicomponent approach to physical activity behavior change has been proposed (Lachman et al., 2018). While theory-based programs are generally successful, optimizable, and adaptable precision medicine approaches will improve cost-efficiency of interventions and health outcomes for individuals who show poor adherence to onesize fits all approaches. That is, predicting from a set of variables, who is more or less likely to adhere to an intervention from the outset will allow the practitioner to provide alternative and individualized interventions prior to individuals having to either demonstrate poor adherence or present with deteriorating health. 
Within the intervention setting, several recent studies have demonstrated that pre-intervention brain structure, known to be critical in supporting executive functions (Burzynska et al., 2011; Tadayon et al., 2020), is associated with individual differences in adherence to exercise interventions. Specifically, regions of the prefrontal, temporal and somatosensory cortices have been associated with adherence to each respective exercise intervention (Best et al., 2017; Gujral et al., 2018). Relatedly, several reviews and observational studies have suggested that the relationship between exercise and the brain is bi-directional (Audiffren \& André, 2019; Cheval, Orsholits, et al., 2020). This hypothesis leverages the concepts of cognitive reserve and brain maintenance (Stern et al., 2020), such that there is a circular nature between greater cognitive and brain resources and higher participation in complex exercise behaviors, which in turn helps with the maintenance and upkeep of cognitive and brain health. While rarely measured directly, proxy measures of brain reserve and brain maintenance include brain structure and brain functional connectivity (for a review see: Stern et al., 2019). In terms of function, numerous applications of functional and anatomical connectivity have led to the observation that the brain is organized into large-scale functional networks (Yeo et al., 2011). A number of these networks, such as the frontoparietal control network (FPCN), the default mode network (DMN) and the dorsal attention network (DAN) change with age (Grady et al., 2016) and are thought to be particularly important in agerelated cognitive decline (Marek \& Dosenbach, 2018; $\mathrm{Ng}$ et al., 2016). These networks also subserve internally and externally directed cognition (Spreng et al., 2013), including broadly defined executive function. Given the heterogeneity of these brain networks and their importance in higher-order cognition, individual patterns of functional connectivity in older adults may be predictive of exercise adherence. Indeed, prior work on mindfulness and meditation have demonstrated that functional connectivity within the default mode network and in frontal and temporal nodes are strong predictors of adherence (Saghayi et al., 2020).

The term "prediction" is used in a number of ways in the literature and can commonly refer to either the correlation of one variable in a group at one point in time with another variable in that same group at another point in time (within-sample correlation) or it can refer to a generalizable model that makes predictions on out-ofsample participants (Gabrieli et al., 2015). Prior studies on psychosocial and behavioral "predictions" of exercise adherence have typically used correlation and therefore their results are likely overly optimistic (Yarkoni \& Westfall, 2017). This overestimation and poor generalizability is compounded further when one considers they only explain relatively small amount of variance $(<20 \%)$ in adherence (Rejeski et al., 2007; Rhodes et al., 2001). Consequently, calls have been made to introduce prediction statistics into psychological and cognitive neuroscience research to improve the generalizability of the results, specifically when using brain-based metrics (Gabrieli et al., 2015). In the case of exercise interventions for example, being able to successfully predict adherence to exercise interventions with generalizable results prior to engagement in the intervention would allow for optimization or individualization of an alternative intervention for those who are predicted to poorly adhere. For example, by providing those who are predicted to adhere poorly with a health coach (Olsen \& Nesbitt, 2010) or just-in-time messaging (Nahum-Shani et al., 2018), paving the way towards efficacious precision medicine approaches in health-based settings, when time can rarely be lost, and cost-efficiency is 
of upmost importance. This is also important as engagement in effective exercise interventions may lead to successful maintenance of physical activity after cessation of the intervention (Madigan et al., 2021) and so predicting adherence to the exercise intervention may also provide insights into sustained exercise behaviors.

In a secondary analysis of data from a randomized control trial of exercise in older adults, the objectives of this study were to predict adherence to a 6-month exercise intervention and an active control in a structured and supervised group-based intervention. We hypothesized that multiple metrics of brain structure and function in frontoparietal control, default mode and dorsal attention networks and regions (prefrontal, temporal, parietal) would predict adherence to the exercise intervention and that these measures would augment traditional behavioral and psychosocial measures.

\section{Methods}

\subsection{Participants}

This study is a secondary analysis of data from participants who participated in a six-month randomized controlled exercise trial (clinical study identifier: NCT01472744, November 16, 2011). The study procedures were approved by the University of Illinois Institutional Review Board and written informed consent was obtained from all participants prior to any research activities. Healthy but low active older adults were recruited in Champaign County, Illinois. Two hundred and forty-seven (169 women) lowactive older adults met inclusion criteria for the initial clinical trial. Of which, 165 underwent structural and functional magnetic resonance imaging (MRI) at baseline and 131 had complete data across all variables and were included in this analysis (see further exclusion criteria based on adherence below). Participants in the initial trial were randomized to one of four intervention conditions; a walking intervention, the same walking intervention plus a dietary supplement designed to enhance lean muscle mass, a dancing intervention and an active control consisting of a stretching and toning intervention. All intervention groups met for approximately one hour, three times per week for six months and for the purpose of this analysis we analyzed our main outcome (adherence) with all four conditions combined. A one-way analysis of variance revealed no significant differences in adherence between the conditions $\left(F_{1,129}=1.11, p=0.294\right)$ and no significant differences in baseline characteristics between conditions existed (supplementary table 1). Further we replicate our results in the most common intervention conditions (walking) only as a sensitivity analysis with a reduced sample size $(n=59)$ to ensure our results were not affected by condition assignment (supplementary material). Initial inclusion criteria included being between the ages of 60 and 80 years old, free from psychiatric and neurological illness including no history of stroke, transient ischemic attack, or head trauma, scored > 23 on the Mini-Mental State Exam, $>21$ on a Telephone Interview of Cognitive Status questionnaire, $<10$ on the Geriatric Depression Scale, at least 75\% right-handed based on the Edinburgh Handedness Questionnaire (a criterion related to functional magnetic resonance imaging (MRI) analyses), demonstrated normal or corrected-to-normal vision of at least 20/40 and no color blindness, screened for safe participation in an MRI environment (e.g., no metallic implants that could interfere with the magnetic field or cause injury and 
no claustrophobia) and reported to have participated in no more than two bouts of moderate exercise per week within the past six months (with the goal of recruiting low active older adults). Table 1 contains complete characterization of the study participants included in this analysis. All methods were carried out in accordance with the Declaration of Helsinki.

\subsection{Adherence}

Our primary outcome, adherence, was modelled as percentage attendance to the weekly intervention sessions. These supervised sessions were scheduled three times per week and lasted approximately for one hour. The number and frequency of the sessions were consistent across all four conditions. For this analysis, participants $(\mathrm{N}=34)$ who dropped-out of the study (i.e., did not complete the intervention) were excluded, as were those who failed to attend at least half of the intervention sessions $(n=12)$ as our aim was to capture and predict variations in adherence to the entire 6month intervention.

\subsection{Psychosocial, physical function and activity, health, and cognitive features}

A comprehensive battery of psychosocial, physical and cognitive assessments was completed by each participant pre-intervention. A complete table of these assessments is found in the supplementary material. Eighty-four assessments were initially included in this analysis. In brief, these assessments included self-reported psychosocial questionnaires gauging participants self-efficacy (global, exercise and gait self-efficacy), leisure time activity, perceived sleep quality, anxiety, depression and selfworth/esteem, barriers to exercise, self-regulation, stress, loneliness and subjective memory. All psychosocial measures were taken at week one or in some instances repeated at week three. A set of physical function tests were also collected at week one, which included a stair climb test, arm curl, sit and reach and back scratch. Seven days of accelerometry capturing objective measures of time spent sedentary, time spent in light or moderate-to-vigorous aerobic physical activity and average daily step counts were also collected. The procedures to capture, preprocess and validate these measures can be found in previous publications (Burzynska et al., 2020; Fanning et al., 2017). A measure of cardiorespiratory fitness from a complete cardiopulmonary exercise test was also included as were measures of body composition (see our previous works (Burzynska et al., 2014; Voss et al., 2016) for a detailed description of the methodology of these measures). Finally, a battery of neuropsychological tasks were completed in week one which included numerous assessments of vocabulary, abstract, inductive and visuospatial reasoning, memory and perceptual speed, taken from the Virginia Cognitive Aging Project (Salthouse \& Ferrer-Caja, 2003). Intervention condition assignment was also included as a feature.

\subsection{Neuroimaging features}

\subsubsection{Magnetic resonance imaging: acquisition}


Participants undertook an MRI scanning session in a three Tesla Siemens Trio Tim system with a 12-channel head coil. High-resolution structural MRI scans were acquired using 3D MPRAGE T1-weighted sequences $(\mathrm{TR}=1900 \mathrm{~ms} ; \mathrm{TE}=2.32 \mathrm{~ms} ; \mathrm{TI}$ : $900 \mathrm{~ms} ;$ flip angle $=9^{\circ} ;$ matrix $=256 \times 256 ; \mathrm{FOV}=230 \mathrm{~mm} ; 192$ slices; resolution $=0.9$ $\times 0.9 \times 0.9 \mathrm{~mm}$; GRAPPA acceleration factor 2 ). T2* ${ }^{*}$-weighted resting state echoplanar imaging (EPI) data was obtained with the following parameters:

$\left(6 \mathrm{~min}, \mathrm{TR}=2 \mathrm{~s}, \mathrm{TE}=25 \mathrm{~ms}\right.$, flipangle $=80^{\circ}, 3.4 \times 3.4 \mathrm{~mm}^{2}$ in-plane resolution, $354 \mathrm{~mm}$-thick slices acquired in ascending order, Grappa acceleration factor $=2,64 \times 64$ matrix). Structural and resting state functional images were acquired with these scanning parameters and the preprocessing and analyses of each respective modality are outlined in the following two sections.

\subsubsection{Structural MRI preprocessing and analyses}

Cortical reconstruction and image segmentation and estimation of the cortical surface models was performed using the freely available FreeSurfer software v.5.3 (http://surfer-nmr.mgh.harvard.edu/). For preprocessing of the cortex a 3dimensional surface model was created using the "recon-all" surface-based stream. Automated Talairach transformation and intensity normalization were followed by nonbrain tissue removal, tessellation of the gray and white matter boundary and automated topology correction. Finally, surface deformation enabled the detection of tissue boundaries; grey-white and grey-CSF borders. The cortical surfaces were then inflated and registered to a spherical atlas that used individual cortical folding patterns to match cortical geometry across participants. Cortical thickness was calculated at each vertex in the cortex as a measure of the distance between the white and pial surfaces and cortical surface area was calculated by averaging the area of all faces that meet at a given vertex on the white matter surface. We chose to analyze cortical thickness and surface area separately given their genetic independence and sensitivity to clinical and aging outcomes (Storsve et al., 2014). Automatic labelling per the Desikan-Killany cortical parcellation scheme was performed and average cortical thickness and cortical surface area were calculated within each parcellation resulting in 136 structural features (68 features per modality) to be used for feature selection.

\subsubsection{Resting state functional connectivity preprocessing and analyses}

Preprocessing of the functional resting state data was performed using the CONN-toolbox v.19c (Whitfield-Gabrieli \& Nieto-Castanon, 2012), relying upon SPM v.12 (Wellcome Department of Imaging Neuroscience, UCL, London, UK) in MATLAB R2019a (The MathWorks Inc, Natick, MA, USA). The default preprocessing pipeline implemented in CONN was performed which consists of the following steps: functional realignment and unwarping, slice timing correction, outlier identification, segmentation (into grey matter, white matter and cerebrospinal fluid tissue) and normalization into standard Montreal Neurologic Institute (MNI) space with $2 \mathrm{~mm}$ isotropic voxels for functional data and $1 \mathrm{~mm}$ for anatomical data, using $4^{\text {th }}$ order spline interpolation. Finally, functional scans were spatially smoothed using a $6 \mathrm{~mm}$ Gaussian kernel. During the outlier detection step, acquisitions with framewise displacement above $0.9 \mathrm{~mm}$ (per 
several prior publications in studies with older adults who are more susceptible to movement within the scanner (Fitzhugh et al., 2019; Pistono et al., 2021; Vieira et al., 2020) or global BOLD signal changes above five standard deviations were flagged as potential outliers using the Artefact Detection Tools (www.nitrc.org/projects/artifact_detect). Two participants were removed from the final analyses for having $>40$ volumes flagged. This cut off was determined based on preserving at least five minutes of scanning time (Van Dijk et al., 2009). Additionally, mean framewise displacement was calculated via the Jenkinson method (Jenkinson et al., 2002) and regressed out of the final analysis (see statistical analysis section below). This was done to be over-conservative given previous studies have shown high degree of motion-behavior correlations (Siegel et al., 2017), despite the fact that no motion variable was significantly correlated with adherence outcomes in our study (all $p>0.1)$. Denoising of the functional data was performed using a component-based correction method, CompCor (Behzadi et al., 2007) and temporal band-pass filtering $(0.01-0.1 \mathrm{~Hz})$ to remove physiological, subject-motion and outlier artefacts. Linear regression was used to remove the effects of these artifacts on the BOLD time series for each voxel and each subject taking into account noise components from voxels within white matter and cerebrospinalfluid, estimated subject-motion parameters (three rotation and three translation parameters and six other parameters representing their first order time derivatives), scrubbing and constant and first-order linear session effects.

To prepare the functional data for feature selection, we parcellated the functional scans into a medium resolution 300 region (node) atlas with a 17-network parcellation scheme (Schaefer et al., 2018). This choice was taken to strike a balance between biological resolution and dimensionality. Mean BOLD activity was calculated within each node and transformed into a 300 by 300 correlation matrix where the timeseries at each node was correlated with that of every other node. This matrix then underwent Fisher's $Z$ transformation. Functional brain connectivity was summarized using degree count, a graph theory metric which represents whole brain connection density for each node, per a previous publication (Vogel et al., 2018). This metric reduces the dimensions of the functional connectivity data into a sparse matrix that better represents real world graphs (Power et al., 2013). Degree count for each participant was calculated at several thresholds using a fixed network cost (keeping the strongest $15 \%, 20 \%$ and $25 \%$ of connections) and a final threshold was chosen through cross-validation (see statistical analysis section). Degree count at each node here represents a single measure of the sum of connections between that node and every other node in the cortex characterizing its degree of connectedness within the cortical gray matter.

\subsection{Power analysis}

We performed a power analysis (in R using the "pwr" package) on our sample size to ensure sufficient power was gained to detect a true effect. Based on our sample size $(\mathrm{N}=131)$ and an assumed type I error rate of 0.05 , we calculated an estimated $96 \%$ power to detect an effect size of 0.22 from a general linear model with 38 covariates (multimodal model). In the reduced sample size of $\mathrm{N}=59$ for the sensitivity analysis (supplementary material) this power fell to $60 \%$ for the same effect size. 


\subsection{Statistical analysis}

To predict adherence to the exercise interventions we used Elastic net regression, a regularized (penalized) regression method within a nested cross-validation procedure. Elastic net aims to avoid overfitting by producing less-complex models via applying both a L1 and L2 norm penalty to calculate the coefficients of each feature. Elastic net is a linear combination of both Ridge regression (L2 norm) and least absolute selection and shrinkage operate (Lasso) regression and as such strikes a balance between shrinking coefficients towards zero (Ridge) and in the case of features that have no predictive value, to exactly zero (Lasso), relative to the maximum likelihood estimates. The predictors with non-zero coefficients are therefore interpreted as those that contained predictive information and contributed to the final model of predicting our outcome. This model is particularly useful in cases where correlated features are present (i.e., neuroimaging features and some psychosocial measures) as the combination of both the L1 and L2 penalties will maintain groups of correlated features in the model (whereas the L1 penalty (Lasso) alone would remove all but one of the correlated features). The amount of shrinkage is determined via tuning of two hyperparameters $\lambda_{1}$ and $\lambda_{2}$. Results of the optimal hyperparameters for each model are found in Table 2. In this analysis we used a nested cross-validation procedure where the dataset is split into a 10-fold outer loop (to evaluate model performance) and a 10fold inner loop (to tune the hyperparameters using grid search within each inner fold). Nested-cross validation avoids optimization bias that simple cross-validation could potentially suffer from when using the same folds to both tune the hyperparameters and test the prediction performance on. In this case, the folds were kept consistent across each model by setting the same random seed and all variables were centered and scaled within each inner loop and applied to the outer folds. First, to select the optimal degree count matrix threshold, models were trained over each threshold density and the model that predicted the left-out outer folds with the smallest root mean squared error (RMSE) was used in the final models. In the final models, a manual feature preselection was performed by removing features that didn't correlate with adherence at $p<0.1$ $(p<0.1$ was selected to maintain only features that contained potentially predictive information) and age, biological sex and mean framewise displacement (functional connectivity only) were regressed out of the features. Three separate models were trained: (1) imaging features only, (2) Psychosocial, physical function and activity, health, and cognitive features only and (3) multimodal features containing all features. In the case of model 2, the combination of nested cross-validation and Elastic net failed to predict adherence in the left-out outer folds, potentially due to the small number of features in the model $(p=6)$ and as such, for the non-imaging model only we ran a simple 10-fold cross-validation model using Ridge regression (L1 norm penalty only). Model performance (prediction on the left-out folds) was calculated as the squared correlation between the predicted and observed values $\left(R^{2}\right)$. Other performance metrics included RMSE, which measures the average prediction error as the average difference between the observed and predicted values and the mean absolute error (MAE) as the average absolute difference between the observed and predicted values. RMSE and MAE are related with MAE being less sensitive to outliers and the lower the value the better the model performance. For $\mathrm{R}^{2}$, a higher value equates to better performance. To 
assess the significance of the $\mathrm{R}^{2}$ prediction performance, 1000 non-parametric permutations were performed, and the p-value of the permutation tests was calculated as the proportion of sampled permutations that are greater or equal to the true prediction correlation. All statistics were performed in RStudio version 3.6.3 using "dplyr", "purr", "penalized" and "pensim" packages. Figures were generated using "ggplot2".

\section{Results}

Table 1 presents demographic details of the participants included in this analysis. Our sample consisted of mostly female, white, and educated participants and the mean adherence was $81 \%$.

Table 1. Participant Characteristics

\begin{tabular}{l|l} 
N & 131 \\
Age (mean (SD)) & $65.79(4.65)$ \\
Gender: Female (\%) & $63(71.6)$ \\
Race (\%) & \\
White & $79(89.7)$ \\
African American or black & $7(8)$ \\
Asian & $2(2.3)$ \\
Years of education (mean (SD)) & $15.89(2.66)$ \\
Adherence (\%) & $81(12)$ \\
\hline
\end{tabular}

Table 2 contains prediction metrics for each modality using Elastic net models and Ridge regression for the cognitive/psychosocial only model. All models resulted in a significant prediction of adherence to the exercise interventions with the multimodal model performing the best, followed by the imaging and then the psychosocial, physical function and activity, health, and cognitive model (Table 2 and Figure 1).

Table 2. Model performance metrics

\begin{tabular}{lcccc}
\hline Model & $\mathbf{R}^{2}$ & RMSE & MAE & P-value \\
Multimodal & 0.22 & 0.10 & 0.09 & $<0.001$ \\
Imaging & 0.15 & 0.11 & 0.09 & $<0.001$ \\
Psych/cog/health & 0.06 & 0.12 & 0.10 & 0.001 \\
\hline
\end{tabular}

Performance metrics derived from nested cross-validation where the optimal hyperparameters for Elastic net regression were tuned in an inner loop and used to predict adherence in the left out outer loop. The $p$-value for each model is derived by comparing the actual $\mathrm{R}^{2}$ against a null distribution derived from 1000 non-parametric permutations. 
Multimodal
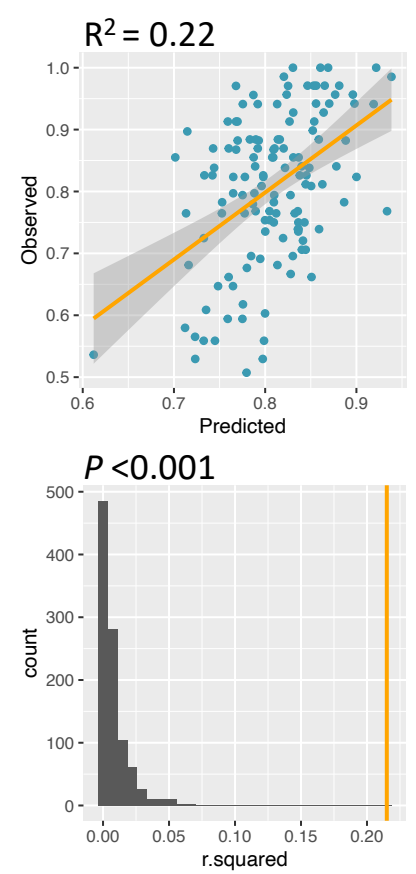

Imaging
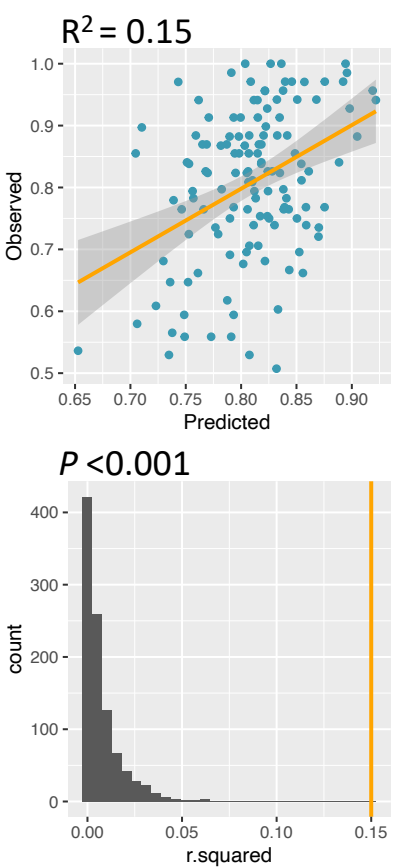

Psych/cog/health
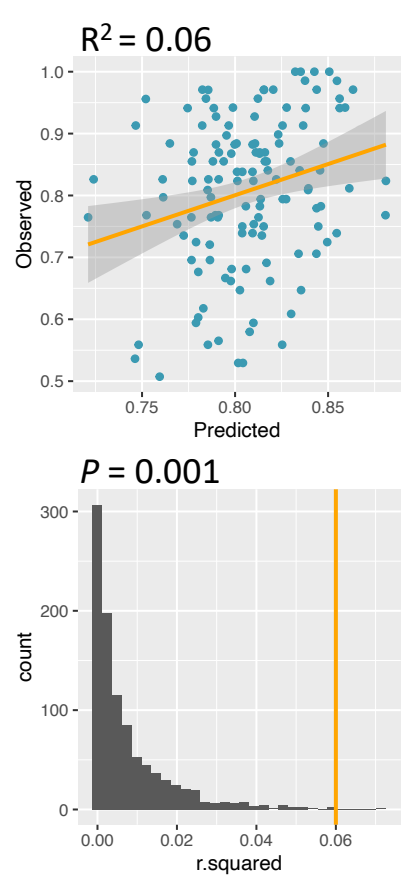

Figure 1. Prediction performance $\left(R^{2}\right)$ of each model (top row) and the significance test ( $p$-value) of each model via 1000 permutations (bottom row). Top row: $R^{2}$ represents the squared correlation between the predicted and observed adherence values via Elastic net regression with nested cross-validation. Bottom row: Null distribution of the prediction performance via 1000 non-parametric permutations. The orange vertical line represents the actual model performance $\left(R^{2}\right)$ and the $p$-value from the permutation tests is calculated as the number of sampled permutations that are greater or equal to the true prediction correlation.

Univariate correlations between those features selected after manual feature preselection and adherence is found in Table 3. Standardized coefficients for each feature selected in each model are found in Table 3. A summary figure of the multimodal model is found in Figure 2. For the functional connectivity features, the result broadly shows multiple distributed negative nodes in the somatomotor, visual, temporoparietal and ventral attention networks and multiple positive nodes distributed across DMN, DAN and FPCN predictive of adherence. For the structural features, distributed features across both hemispheres which were almost all positive for cortical thickness and negative for surface area were found to predict adherence. For the Psych/cog/health features, several psychosocial questionnaires (i.e., gait and walking self-efficacy, stress and sleep quality), biological sex and one neuropsychological test (i.e., letter comparison) were selected as predictive features.

Table 3. Univariate correlations and Elastic net feature selection

\begin{tabular}{lllllllrrr} 
Feature & $r$ & $P$ & \multicolumn{2}{c}{ Psych/cog/health } & \multicolumn{2}{c}{ Imaging } & \multicolumn{2}{c}{ Multimodal } \\
& & & $\lambda 1$ & $\lambda 2$ & $\lambda 1$ & $\lambda 2$ & $\lambda 1$ & $\lambda 2$ \\
Ih_postcentral_SMb & & & n/a & 100 & 0.173 & 100 & 0.194 & 89 \\
& -0.147 & 0.094 & & & & -0.0076 & -0.0069
\end{tabular}




\begin{tabular}{|c|c|c|c|c|c|}
\hline Ih_superiorparietal_DANa & 0.154 & 0.079 & & 0.0058 & 0.0061 \\
\hline Ih_precuneus_FPCNc & 0.148 & 0.091 & & 0 & 0 \\
\hline Ih_lateralPFC_DMNb & 0.180 & 0.039 & & 0.0057 & 0.0056 \\
\hline Ih_vPFC_DMNb & 0.170 & 0.052 & & 0.0069 & 0.0061 \\
\hline Ih_temporalparietal & -0.184 & 0.035 & & -0.0081 & -0.0081 \\
\hline rh_extrastriateinf_perivisual & -0.177 & 0.043 & & -0.0054 & -0.0048 \\
\hline rh_extrastriateinf_perivisual & -0.145 & 0.098 & & 0 & 0.0004 \\
\hline rh_postcentral_SMa & -0.145 & 0.098 & & 0.0013 & 0.0014 \\
\hline rh_S2_SMb & -0.179 & 0.040 & & -0.0027 & -0.0025 \\
\hline rh_parietaloccipital_DANa & 0.185 & 0.034 & & 0.0109 & 0.0105 \\
\hline rh_parietaloperculum_VANa & -0.155 & 0.077 & & -0.0079 & -0.0085 \\
\hline rh_parietaloperculum_VANa & -0.177 & 0.043 & & -0.0008 & -0.0012 \\
\hline rh_frontalmedial_VANa & 0.181 & 0.039 & & 0.0070 & 0.0054 \\
\hline rh_temporal_FPCNa & 0.164 & 0.061 & & 0.0031 & 0.0035 \\
\hline rh_mPFC_DMNa & 0.196 & 0.025 & & 0.0059 & 0.0063 \\
\hline rh_temporalparietal & -0.204 & 0.019 & & -0.0004 & -0.0001 \\
\hline rh_temporalparietal & -0.221 & 0.011 & & -0.0106 & -0.0112 \\
\hline Ih_cuneus_thickness & -0.161 & 0.066 & & -0.0119 & -0.0126 \\
\hline Ih_entorhinal_thickness & 0.178 & 0.042 & & 0.0083 & 0.0068 \\
\hline Ih_parsorbitalis_thickness & 0.164 & 0.062 & & 0.0033 & 0.0033 \\
\hline Ih_postcentral_thickness & 0.148 & 0.092 & & 0.0089 & 0.0097 \\
\hline rh_inferiortemporal_thickness & 0.149 & 0.090 & & 0.0081 & 0.0076 \\
\hline rh_isthmuscingulate_thickness & -0.167 & 0.056 & & -0.0113 & -0.0105 \\
\hline rh_frontalpole_thickness & 0.147 & 0.095 & & 0.0021 & 0.0025 \\
\hline Ih_cuneus_area & -0.148 & 0.091 & & -0.0001 & 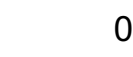 \\
\hline Ih_lingual_area & -0.150 & 0.087 & & -0.0051 & -0.0040 \\
\hline Ih_parsorbitalis_area & -0.170 & 0.053 & & -0.0075 & -0.0070 \\
\hline Ih_pericalcarine_area & -0.178 & 0.042 & & -0.0050 & -0.0044 \\
\hline rh_lateraloccipital_area & -0.158 & 0.071 & & -0.0019 & -0.0004 \\
\hline rh_transversetemporal_area & -0.148 & 0.092 & & -0.0081 & -0.0084 \\
\hline Self-efficacy for walking (w3) & -0.165 & 0.060 & -0.0006 & & -0.0078 \\
\hline Gait self-efficacy (w1) & 0.185 & 0.035 & 0.0009 & & 0.0068 \\
\hline Perceived stress scale & -0.153 & 0.081 & -0.0012 & & -0.0063 \\
\hline Perceived sleep quality index & -0.187 & 0.032 & -0.0025 & & -0.0049 \\
\hline Biological sex & -0.258 & 0.003 & -0.0351 & & -0.0165 \\
\hline Letter comparison task & -0.156 & 0.074 & -0.0048 & & -0.0061 \\
\hline
\end{tabular}

Imaging feature names are constructed as follows: 'hemishere_region_network_or_structural measure'. Network is derived from the 17-network cortical parcellation from Yeo et al., 2011 via automatic labeling using the Schaefer 300 atlas. $r$ and $P$ represent univariate Pearson's correlation. Coefficients from each prediction model represent the standardized and penalized coefficient used to predict Adherence. 0 means that the feature was effectivity removed from the model due to having little or no predictive value. $\lambda 1$ and $\lambda 2$ represent the optimal cross-validated hyperparameters for each model. $\lambda 1$ is "n/a" for 


\begin{abstract}
the psych/cog/health model as simple cross-validation and Ridge regression (L2 norm penalty only) was used to predict adherence as the Elastic net with nested cross validation failed to find a solution to predict adherence with these features only. Biological sex was coded as 2 for women and 1 for men (i.e., a negative coefficient means that men adhered better than women).
\end{abstract}
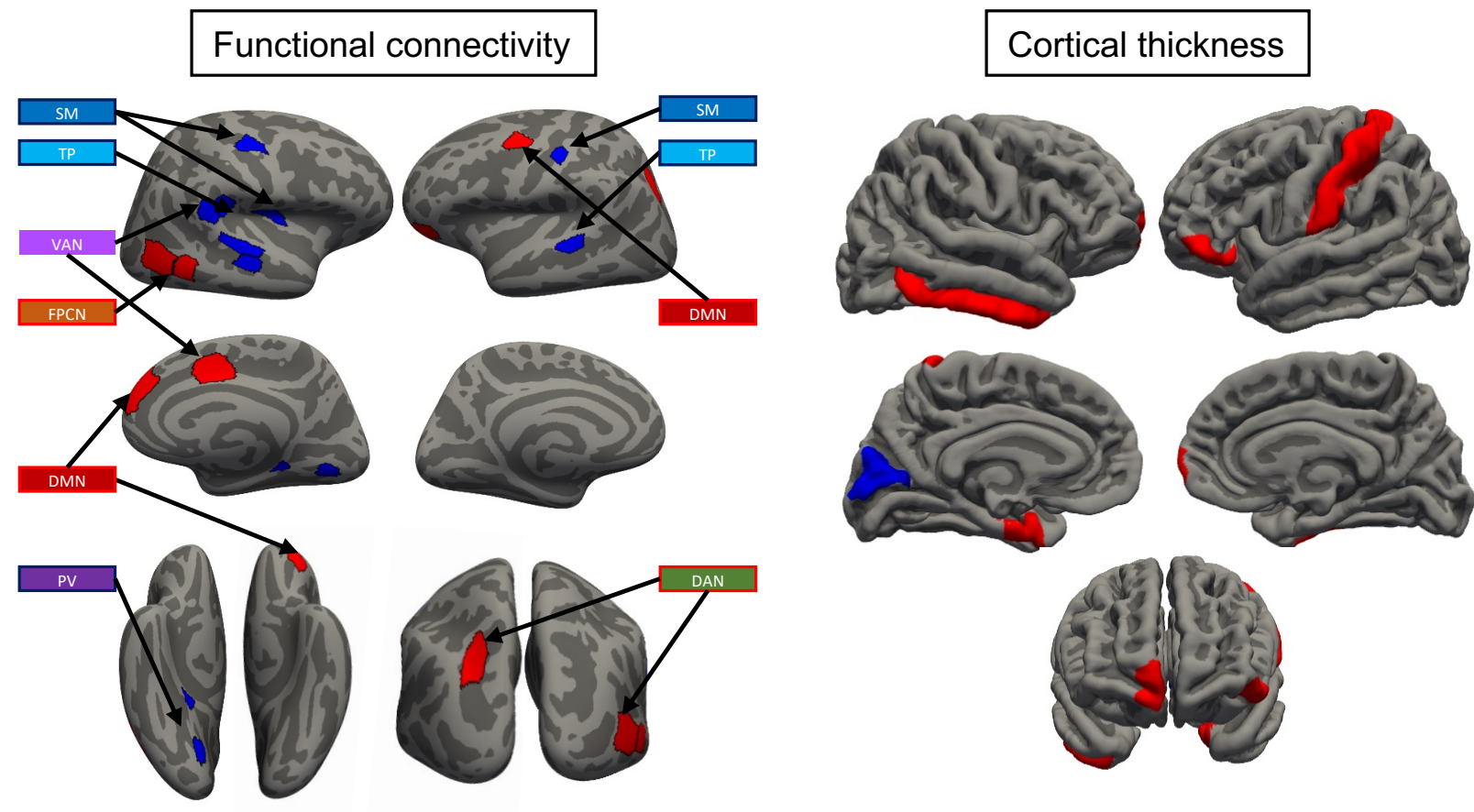

\title{
Surface area
}
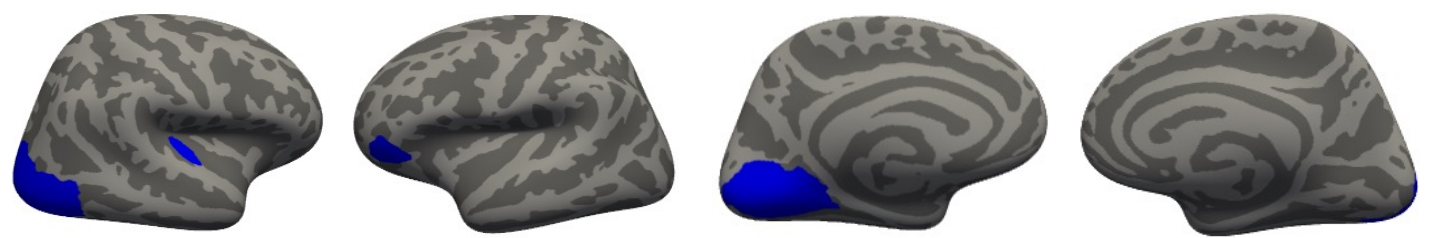

Figure 2. Summary figure depicting the selected features of brain structure and function in the multimodal model (best performing model). For the functional connectivity features (top left), each feature represents degree count (number of connections that each node has with every other node in the cortical parcellation). That is, red features (positive nodes) are interpreted as the higher the connectivity between that node and every other node in the cortex, the higher the adherence. Blue features (negative nodes) suggest that less connections between these nodes and the rest of the brain the higher the adherence. Mapping these regions to intrinsic resting state functional networks (17 Yeo networks via the Schaefer 300 atlas) reveals a broad pattern of positive nodes in the DMN, DAN and FPCN and negative nodes in primary information processing networks. In general, greater cortical thickness and lower cortical surface area in several disperse brain regions were predictive of adherence.

\section{Discussion}

In this study we demonstrated that brain-based measures of functional connectivity, cortical thickness and surface area predicted future adherence to a structured groupbased exercise intervention in older adults. Using a machine learning framework that applies penalized regression with cross-validation we also replicated earlier studies indicating that aspects of self-efficacy and biological sex are predictive of adherence to exercise and that together, multimodal features provide the strongest predictive value. 
Currently, our model performance $\left(R^{2}\right.$ of .22) remains far from having utility in a clinical setting. While we replicated the finding that traditional psychosocial and demographic measures predict adherence to exercise, the ability of neuroimaging features alone to predict adherence to a structured group-based exercise intervention suggests substantial utility of these measures for future research into precision medicine and adaptive intervention approaches. Additionally, the increase in model performance when combined suggests that multimodal features provide independent relevant information in the prediction of exercise adherence.

In a research setting, the application of prediction modelling could aid in the development of adaptive intervention strategies. For example, in those who can successfully engage in a theory-based intervention, positive results have been shown. However, successful engagement in long-term interventions is non-trivial and not all individuals will adhere. If one could predict these individuals from the outset, redeployment of resources to tailor the intervention in these individuals with the goal of improving adherence and subsequent efficacy could be done. For example, our results could be leveraged in future prospective studies to test whether adaptive interventions based on pre-intervention characteristic and brain signatures (our predictions) result in better adherence and intervention efficacy. Additionally, successful engagement in an exercise intervention may also be related to long-term maintenance of exercise behaviors beyond the intervention (Madigan et al., 2021). Consequently, applying this type of prediction modeling to recent large population and cohort studies (e.g., UK Biobank or Human Connectome Project) with imaging and self-report physical activity data could help one determine the proportion and characteristics of individuals who would require additional tailoring when designing future exercise interventions.

Regarding our imaging analysis, we took a whole-brain approach to the prediction of exercise. Our results however could be leveraged to validate these results in future hypothesis-driven studies. For example, nodes with high global functional connectivity predictive of adherence in our study were found in the DMN, DAN and FPCN, three related networks that contribute to internally and externally directed cognition (Spreng et al., 2013) and in the implementation of executive control processes to maintain goals and inhibit distractions, respectively (Spreng et al., 2010; Vincent et al., 2008). Similarly, cortical structure in postcentral, ventrolateral/orbitofrontal, and inferior temporal regions have been shown to be associated with executive functions, especially in healthy aging (Burzynska et al., 2011). As such, direct study of the interplay between these networks at rest or during executive function tasks that engage these networks may provide stronger predictive utility.

Numerous randomized controlled trials of exercise in aging have demonstrated that exercise can improve (with mixed effect sizes) cognitive function and positively effect brain structure and functional connectivity (Erickson et al., 2019; Gomes-Osman et al., 2018; Northey et al., 2018). Yet the hypothesized bi-directional that the relationship between exercise and the brain and cognition is starting to be tested (Cheval, Orsholits, et al., 2020). In our current study, individual differences in proxy measures of cognitive and brain reserve (functional connectivity nodes, cortical thickness and surface area, respectively) within primary information processing networks and regions were predictive of exercise adherence. Prior exercise interventions have shown interventionmediated increases in the functional connectivity of somatosensory networks in older 
adults (McGregor et al., 2018). Our results therefore potentially provide supportive evidence in favor of the hypothesis that the relationship between exercise and the brain is bi-directional (Loprinzi et al., 2013; Ponce \& Loprinzi, 2018). Notwithstanding, our prediction models need to be tested in prospective and experimental studies to conclude a causal association.

Several limitations to this study mean that our results should be interpreted with reasonable caution. Firstly, the complexity of adherence to a 6-month exercise intervention may not be completely captured in a single variable representing the percentage attendance to the intervention settings and so additional explained variance not captured by our measures may be due to other reasons such as scheduling conflicts related to family, work or breaks due to discomfort. Second, it is of note that inferential guarantees regarding variable estimates (coefficients) in penalized regression models cannot be made. I.e., traditional p-values or confidence intervals for each estimate do not exist (Lockhart et al., 2014). Therefore, estimates produced by the elastic net model are biased and should not be interpreted as the population parameter. While advances in post-selection inference methods for the Lasso have been made ((Lee et al., 2016; Lockhart et al., 2014) which allow for more valid confidence intervals and significance testing for Lasso estimates), such methods do not exist for Elastic Net, the use which was important in our case given the collinearity of the predictors in our study. Third, our sample size was relatively small for machine learning. As such, testing on completely left-out subjects (test-train splits) was not feasible and so we attempted to perform the most rigorous and generalizable approach possible via nested cross-validation. Fourth, given the difficulty and costs associated with this type of research and the vast number of measures that were available in this unique dataset, replication of this prediction in a completely independent validation dataset is unavailable at this moment. Fifth, our participants were homogenous. Several factors have recently been highlighted as a reason why global physical inactivity rates continue to be low (Pratt et al., 2020) with one being that most of the research on understanding physical activity behaviors has occurred in high-income countries and so more work in diverse populations and lowincome countries is needed to fully generalize this type of prediction. Lastly, our battery of neuropsychological tasks included executive function tests that measured constructs such as abstract, inductive and visuospatial reasoning rather than other executive tasks like inhibitory control that may be more related to exercise adherence (Cheval, Daou, et al., 2020).

Our results showed that the combination of psychosocial, cognitive and demographic, and multimodal imaging metrics can predict adherence to an exercise intervention in older adults and provide independent relevant predictive value. Prospective testing of these predictions and their validation will allow researchers and eventually clinicians to leverage them through personalized medicine approaches.

\section{Acknowledgments}

We would like to thank, Anya Knecht, Susan Houseworth, Nancy Dodge, Hilly Tracy, Robert Weisshappel and all of the Lifelong Brain and Cognition and Exercise Psychology Laboratory graduate students and staff for their help in participant recruitment and data collection. 


\section{Funding}

This work weas supported by the National Institute on Aging at the National Institutes of Health (R37 AG025667).

\section{Author contributions}

TPM- Conceptualization, design, analysis, interpretation of data, manuscript writing, $A B$ - data acquisition, substantial revision, MV- data acquisition, substantial revision, JFdata acquisition, substantial revision, ES - data acquisition, substantial revision, RPdata acquisition, substantial revision, NG- data acquisition, substantial revision, SWGanalysis, interpretation of data, $\mathrm{CH}$ - design, substantial revision, EMConceptualization, study design, substantial revision, AF- Conceptualization, study design, analysis, interpretation of data, substantial revision

\section{Conflict of interest}

No authors declare any conflict of interest.

\section{Ethics approval}

The University of Illinois Institutional Review Board approved all procedures used in the study.

\section{Consent to participate}

All participants gave written informed consent before participation in any study procedures, all of which conformed to the Declaration of Helsinki for research involving human subjects.

\section{Consent for publication and author responsibilities}

All authors agree to the contents of this manuscript and give consent for its publication.

\section{Availability of data and materials}

All data will be provided upon reasonable request to the corresponding author, without reservation.

\section{References}

Andersen, Mota, \& Pietro. (2016). Update on the global pandemic of physical inactivity. Lancet, 388(10051), 1255-1256. https://doi.org/10.1016/s0140-6736(16)30960-6

Arkkukangas, M., Söderlund, A., Eriksson, S., \& Johansson, A.-C. (2018). One-Year Adherence to the Otago Exercise Program With or Without Motivational Interviewing in Community-Dwelling Older Adults. Journal of Aging \& Physical Activity, 26(3), 390-395. 
Audiffren, M., \& André, N. (2019). The exercise-cognition relationship: A virtuous circle. Journal of Sport and Health Science, 8(4), 339-347.

https://doi.org/10.1016/j.jshs.2019.03.001

Behzadi, Y., Restom, K., Liau, J., \& Liu, T. T. (2007). A component based noise correction method (CompCor) for BOLD and perfusion based fMRI. Neurolmage, 37(1), 90-101. https://doi.org/10.1016/j.neuroimage.2007.04.042

Best, J. R., Chiu, B. K., Hall, P. A., \& Liu-Ambrose, T. (2017). Larger Lateral Prefrontal Cortex Volume Predicts Better Exercise Adherence Among Older Women: Evidence From Two Exercise Training Studies. The Journals of Gerontology: Series A, 72(6), 804-810. https://doi.org/10.1093/gerona/glx043

Brand, R., \& Cheval, B. (2019). Theories to Explain Exercise Motivation and Physical Inactivity: Ways of Expanding Our Current Theoretical Perspective. Frontiers in Psychology, 10. https://doi.org/10.3389/fpsyg.2019.01147

Burzynska, A. Z., Chaddock-Heyman, L., Voss, M. W., Wong, C. N., Gothe, N. P., Olson, E. A., Knecht, A., Lewis, A., Monti, J. M., Cooke, G. E., Wojcicki, T. R., Fanning, J., Chung, H. D., Awick, E., McAuley, E., \& Kramer, A. F. (2014). Physical Activity and Cardiorespiratory Fitness Are Beneficial for White Matter in Low-Fit Older Adults. PLOS ONE, 9(9), e107413. https://doi.org/10.1371/journal.pone.0107413

Burzynska, A. Z., Nagel, I. E., Preuschhof, C., Gluth, S., Bäckman, L., Li, S., Lindenberger, U., \& Heekeren, H. R. (2011). Cortical thickness is linked to executive functioning in adulthood and aging. Human Brain Mapping, 33(7), 1607-1620. https://doi.org/10.1002/hbm.21311

Burzynska, A. Z., Voss, M. W., Fanning, J., Salerno, E. A., Gothe, N. P., McAuley, E., \& Kramer, A. F. (2020). Sensor-measured sedentariness and physical activity are differentially related to fluid and crystallized abilities in aging. Psychology and Aging, 35(8), 1154-1169. https://doi.org/10.1037/pag0000580

Cadmus-Bertram, L., Irwin, M., Alfano, C., Campbell, K., Duggan, C., Foster-Schubert, K., Wang, C.-Y., \& McTiernan, A. (2014). Predicting Adherence of Adults to a 12Month Exercise Intervention. Journal of Physical Activity and Health, 11(7), 1304-1312. https://doi.org/10.1123/jpah.2012-0258

Cheval, B., Daou, M., Cabral, D. A. R., Bacelar, M. F. B., Parma, J. O., Forestier, C., Orsholits, D., Sander, D., Boisgontier, M. P., \& Miller, M. W. (2020). Higher inhibitory control is required to escape the innate attraction to effort minimization. Psychology of Sport and Exercise, 51, 101781. https://doi.org/10.1016/j.psychsport.2020.101781

Cheval, B., Orsholits, D., Sieber, S., Courvoisier, D., Cullati, S., \& Boisgontier, M. P. (2020). Relationship between decline in cognitive resources and physical activity. Health Psychology. http://dx.doi.org.ezproxy.neu.edu/10.1037/hea0000857

de Bruin, M., Sheeran, P., Kok, G., Hiemstra, A., Prins, J. M., Hospers, H. J., \& van Breukelen, G. J. P. (2012). Self-regulatory processes mediate the intentionbehavior relation for adherence and exercise behaviors. Health Psychology, 31(6), 695-703. https://doi.org/10.1037/a0027425

Ding, D., Kolbe-Alexander, T., Nguyen, B., Katzmarzyk, P. T., Pratt, M., \& Lawson, K. D. (2017). The economic burden of physical inactivity: A systematic review and 
critical appraisal. British Journal of Sports Medicine, 51(19), 1392-1409.

https://doi.org/10.1136/bjsports-2016-097385

Ding, D., Lawson, K. D., Kolbe-Alexander, T. L., Finkelstein, E. A., Katzmarzyk, P. T., Mechelen, W. van, \& Pratt, M. (2016). The economic burden of physical inactivity: A global analysis of major non-communicable diseases. The Lancet, 388(10051), 1311-1324. https://doi.org/10.1016/S0140-6736(16)30383-X

Dishman, R. K., Jackson, A. S., \& Bray, M. S. (2014). Self-regulation of Exercise Behavior in the TIGER Study. Annals of Behavioral Medicine, 48(1), 80-91. https://doi.org/10.1007/s12160-013-9573-8

Du, Y., Liu, B., Sun, Y., Snetselaar, L. G., Wallace, R. B., \& Bao, W. (2019). Trends in Adherence to the Physical Activity Guidelines for Americans for Aerobic Activity and Time Spent on Sedentary Behavior Among US Adults, 2007 to 2016. JAMA Network Open, 2(7), e197597-e197597. https://doi.org/10.1001/jamanetworkopen.2019.7597

Erickson, K. I., Hillman, C., Stillman, C. M., Ballard, R. M., Bloodgood, B., Conroy, D. E., Macko, R., Marquez, D. X., Petruzzello, S. J., Powell, K. E., \& Committee*, F. 2018 P. A. G. A. (2019). Physical Activity, Cognition, and Brain Outcomes: A Review of the 2018 Physical Activity Guidelines. Medicine \& Science in Sports \& Exercise, 51(6), 1242-1251. https://doi.org/10.1249/MSS.0000000000001936

Fanning, J., Porter, G., Awick, E. A., Ehlers, D. K., Roberts, S. A., Cooke, G., Burzynska, A. Z., Voss, M. W., Kramer, A. F., \& McAuley, E. (2017). Replacing sedentary time with sleep, light, or moderate-to-vigorous physical activity: Effects on self-regulation and executive functioning. Journal of Behavioral Medicine, 40(2), 332-342. https://doi.org/10.1007/s10865-016-9788-9

Fitzhugh, M. C., Hemesath, A., Schaefer, S. Y., Baxter, L. C., \& Rogalsky, C. (2019). Functional Connectivity of Heschl's Gyrus Associated With Age-Related Hearing Loss: A Resting-State fMRI Study. Frontiers in Psychology, 0. https://doi.org/10.3389/fpsyg.2019.02485

Flegal, K., Kishiyama, S., Zajdel, D., Haas, M., \& Oken, B. (2007). Adherence to yoga and exercise interventions in a 6-month clinical trial. BMC Complementary and Alternative Medicine, 7(1), 37. https://doi.org/10.1186/1472-6882-7-37

Gabrieli, J. D. E., Ghosh, S. S., \& Whitfield-Gabrieli, S. (2015). Prediction as a Humanitarian and Pragmatic Contribution from Human Cognitive Neuroscience. Neuron, 85(1), 11-26. https://doi.org/10.1016/j.neuron.2014.10.047

Garcia, A. W., \& King, A. C. (1991). Predicting Long-term Adherence to Aerobic Exercise: A Comparison of Two Models. Journal of Sport and Exercise Psychology, 13(4), 394-410. https://doi.org/10.1123/jsep.13.4.394

Gomes-Osman, J., Cabral, D. F., Morris, T. P., Mclnerney, K., Cahalin, L. P., Rundek, T., Oliveira, A., \& Pascual-Leone, A. (2018). Exercise for cognitive brain health in aging: A systematic review for an evaluation of dose. Neurology: Clinical Practice, 8(3), 257-265. https://doi.org/10.1212/CPJ.0000000000000460

Grady, C., Sarraf, S., Saverino, C., \& Campbell, K. (2016). Age differences in the functional interactions among the default, frontoparietal control, and dorsal attention networks. Neurobiology of Aging, 41, 159-172. https://doi.org/10.1016/j.neurobiolaging.2016.02.020 
Gujral, S., McAuley, E., Oberlin, L. E., Kramer, A. F., \& Erickson, K. I. (2018). Role of Brain Structure in Predicting Adherence to a Physical Activity Regimen.

Psychosomatic Medicine, 80(1), 69. https://doi.org/10.1097/PSY.0000000000000526

Guthold, R., Stevens, G. A., Riley, L. M., \& Bull, F. C. (2018). Worldwide trends in insufficient physical activity from 2001 to 2016: A pooled analysis of 358 population-based surveys with 1.9 million participants. The Lancet Global Health, 6(10), e1077-e1086. https://doi.org/10.1016/S2214-109X(18)30357-7

Jenkinson, M., Bannister, P., Brady, M., \& Smith, S. (2002). Improved optimization for the robust and accurate linear registration and motion correction of brain images. Neurolmage, 17(2), 825-841. https://doi.org/10.1016/s1053-8119(02)91132-8

Kelly, M. E., Loughrey, D., Lawlor, B. A., Robertson, I. H., Walsh, C., \& Brennan, S. (2014). The impact of exercise on the cognitive functioning of healthy older adults: A systematic review and meta-analysis. Ageing Research Reviews, 16, 12-31. https://doi.org/10.1016/j.arr.2014.05.002

Kohl, H. W., Craig, C. L., Lambert, E. V., Inoue, S., Alkandari, J. R., Leetongin, G., \& Kahlmeier, S. (2012). The pandemic of physical inactivity: Global action for public health. The Lancet, 380(9838), 294-305. https://doi.org/10.1016/S01406736(12)60898-8

Kramer, A. F., Colcombe, S. J., McAuley, E., Eriksen, K. I., Scalf, P., Jerome, G. J., Marquez, D. X., Elavsky, S., \& Webb, A. G. (2003). Enhancing brain and cognitive function of older adults through fitness training. Journal of Molecular Neuroscience: MN, 20(3), 213-221. https://doi.org/10.1385/JMN:20:3:213

Lachman, M. E., Lipsitz, L., Lubben, J., Castaneda-Sceppa, C., \& Jette, A. M. (2018). When Adults Don't Exercise: Behavioral Strategies to Increase Physical Activity in Sedentary Middle-Aged and Older Adults. Innovation in Aging, 2(1). https://doi.org/10.1093/geroni/igy007

Lee, J. D., Sun, D. L., Sun, Y., \& Taylor, J. E. (2016). Exact post-selection inference, with application to the lasso. The Annals of Statistics, 44(3), 907-927. https://doi.org/10.1214/15-AOS1371

Lockhart, R., Taylor, J., Tibshirani, R. J., \& Tibshirani, R. (2014). A SIGNIFICANCE TEST FOR THE LASSO. Annals of Statistics, 42(2), 413-468. https://doi.org/10.1214/13-AOS1175

Loprinzi, P. D., Herod, S. M., Cardinal, B. J., \& Noakes, T. D. (2013). Physical activity and the brain: A review of this dynamic, bi-directional relationship. Brain Research, 1539, 95-104. https://doi.org/10.1016/j.brainres.2013.10.004

Madigan, C. D., Fong, M., Howick, J., Kettle, V., Rouse, P., Hamilton, L., Roberts, N., Gomersall, S. R., \& Daley, A. J. (2021). Effectiveness of interventions to maintain physical activity behavior (device-measured): Systematic review and metaanalysis of randomized controlled trials. Obesity Reviews, n/a(n/a), e13304. https://doi.org/10.1111/obr.13304

Marek, S., \& Dosenbach, N. U. F. (2018). The frontoparietal network: Function, electrophysiology, and importance of individual precision mapping. Dialogues in Clinical Neuroscience, 20(2), 133-140.

McAuley, E., Mullen, S. P., Szabo, A. N., White, S. M., Wójcicki, T. R., Mailey, E. L., Gothe, N. P., Olson, E. A., Voss, M., Erickson, K., Prakash, R., \& Kramer, A. F. 
(2011). Self-Regulatory Processes and Exercise Adherence in Older Adults: Executive Function and Self-Efficacy Effects. American Journal of Preventive Medicine, 41(3), 284-290. https://doi.org/10.1016/j.amepre.2011.04.014

McGregor, K. M., Crosson, B., Krishnamurthy, L. C., Krishnamurthy, V., Hortman, K., Gopinath, K., Mammino, K. M., Omar, J., \& Nocera, J. R. (2018). Effects of a 12Week Aerobic Spin Intervention on Resting State Networks in Previously Sedentary Older Adults. Frontiers in Psychology, 9. https://doi.org/10.3389/fpsyg.2018.02376

Nahum-Shani, I., Smith, S. N., Spring, B. J., Collins, L. M., Witkiewitz, K., Tewari, A., \& Murphy, S. A. (2018). Just-in-Time Adaptive Interventions (JITAIs) in Mobile Health: Key Components and Design Principles for Ongoing Health Behavior Support. Annals of Behavioral Medicine, 52(6), 446-462. https://doi.org/10.1007/s12160-016-9830-8

Ng, K. K., Lo, J. C., Lim, J. K. W., Chee, M. W. L., \& Zhou, J. (2016). Reduced functional segregation between the default mode network and the executive control network in healthy older adults: A longitudinal study. Neurolmage, 133, 321-330. https://doi.org/10.1016/j.neuroimage.2016.03.029

Northey, J. M., Cherbuin, N., Pumpa, K. L., Smee, D. J., \& Rattray, B. (2018). Exercise interventions for cognitive function in adults older than 50: A systematic review with meta-analysis. British Journal of Sports Medicine, 52(3), 154-160. https://doi.org/10.1136/bjsports-2016-096587

Olsen, J. M., \& Nesbitt, B. J. (2010). Health Coaching to Improve Healthy Lifestyle Behaviors: An Integrative Review. American Journal of Health Promotion, 25(1), e1-e12. https://doi.org/10.4278/ajhp.090313-LIT-101

Pistono, A., Guerrier, L., Péran, P., Rafiq, M., Giméno, M., Bézy, C., Pariente, J., \& Jucla, M. (2021). Increased functional connectivity supports language performance in healthy aging despite gray matter loss. Neurobiology of Aging, 98, 52-62. https://doi.org/10.1016/j.neurobiolaging.2020.09.015

Ponce, P., \& Loprinzi, P. D. (2018). A bi-directional model of exercise and episodic memory function. Medical Hypotheses, 117, 3-6. https://doi.org/10.1016/j.mehy.2018.05.020

Power, J. D., Schlaggar, B. L., Lessov-Schlaggar, C. N., \& Petersen, S. E. (2013). Evidence for Hubs in Human Functional Brain Networks. Neuron, 79(4), 798813. https://doi.org/10.1016/j.neuron.2013.07.035

Pratt, M., Varela, A. R., Salvo, D., lii, H. W. K., \& Ding, D. (2020). Attacking the pandemic of physical inactivity: What is holding us back? British Journal of Sports Medicine, 54(13), 760-762. https://doi.org/10.1136/bjsports-2019-101392

Rejeski, W. J., Miller, M. E., King, A. C., Studenski, S. A., Katula, J. A., Fielding, R. A., Glynn, N. W., Walkup, M. P., Ashmore, J. A., \& LIFE Investigators. (2007). Predictors of adherence to physical activity in the Lifestyle Interventions and Independence for Elders pilot study (LIFE-P). Clinical Interventions in Aging, 2(3), 485-494.

Rhodes, R. E., Martin, A. D., \& Taunton, J. E. (2001). Temporal relationships of selfefficacy and social support as predictors of adherence in a 6-month strengthtraining program for older women. Perceptual and Motor Skills, 93(3), 693-703. https://doi.org/10.2466/pms.2001.93.3.693 
Saghayi, M., Greenberg, J., O’Grady, C., Varno, F., Hashmi, M. A., Bracken, B., Matwin, S., Lazar, S. W., \& Hashmi, J. A. (2020). Brain network topology predicts participant adherence to mental training programs. Network Neuroscience, 1-36. https://doi.org/10.1162/netn_a_00136

Salthouse, T. A., \& Ferrer-Caja, E. (2003). What needs to be explained to account for age-related effects on multiple cognitive variables? Psychology and Aging, 18(1), 91-110. https://doi.org/10.1037/0882-7974.18.1.91

Schaefer, A., Kong, R., Gordon, E. M., Laumann, T. O., Zuo, X.-N., Holmes, A. J., Eickhoff, S. B., \& Yeo, B. T. T. (2018). Local-Global Parcellation of the Human Cerebral Cortex from Intrinsic Functional Connectivity MRI. Cerebral Cortex (New York, N.Y.: 1991), 28(9), 3095-3114. https://doi.org/10.1093/cercor/bhx179

Siegel, J. S., Mitra, A., Laumann, T. O., Seitzman, B. A., Raichle, M., Corbetta, M., \& Snyder, A. Z. (2017). Data Quality Influences Observed Links Between Functional Connectivity and Behavior. Cerebral Cortex (New York, N.Y.: 1991), 27(9), 4492-4502. https://doi.org/10.1093/cercor/bhw253

Spreng, R. N., Sepulcre, J., Turner, G. R., Stevens, W. D., \& Schacter, D. L. (2013). Intrinsic Architecture Underlying the Relations among the Default, Dorsal Attention, and Frontoparietal Control Networks of the Human Brain. Journal of Cognitive Neuroscience, 25(1), 74-86. https://doi.org/10.1162/jocn_a_00281

Spreng, R. N., Stevens, W. D., Chamberlain, J. P., Gilmore, A. W., \& Schacter, D. L. (2010). Default network activity, coupled with the frontoparietal control network, supports goal-directed cognition. Neurolmage, 53(1), 303-317. https://doi.org/10.1016/j.neuroimage.2010.06.016

Stern, Y., Arenaza-Urquijo, E. M., Bartrés-Faz, D., Belleville, S., Cantilon, M., Chetelat, G., Ewers, M., Franzmeier, N., Kempermann, G., Kremen, W. S., Okonkwo, O., Scarmeas, N., Soldan, A., Udeh-Momoh, C., Valenzuela, M., Vemuri, P., \& Vuoksimaa, E. (2020). Whitepaper: Defining and investigating cognitive reserve, brain reserve, and brain maintenance. Alzheimer's \& Dementia, 16(9), 13051311. https://doi.org/10.1016/j.jalz.2018.07.219

Stern, Y., Barnes, C. A., Grady, C., Jones, R. N., \& Raz, N. (2019). Brain reserve, cognitive reserve, compensation, and maintenance: Operationalization, validity, and mechanisms of cognitive resilience. Neurobiology of Aging, 83, 124-129. https://doi.org/10.1016/j.neurobiolaging.2019.03.022

Storsve, A. B., Fjell, A. M., Tamnes, C. K., Westlye, L. T., Overbye, K., Aasland, H. W., \& Walhovd, K. B. (2014). Differential Longitudinal Changes in Cortical Thickness, Surface Area and Volume across the Adult Life Span: Regions of Accelerating and Decelerating Change. Journal of Neuroscience, 34(25), 8488-8498. https://doi.org/10.1523/JNEUROSCI.0391-14.2014

Tadayon, E., Pascual-Leone, A., \& Santarnecchi, E. (2020). Differential Contribution of Cortical Thickness, Surface Area, and Gyrification to Fluid and Crystallized Intelligence. Cerebral Cortex, 30(1), 215-225. https://doi.org/10.1093/cercor/bhz082

Van Dijk, K. R. A., Hedden, T., Venkataraman, A., Evans, K. C., Lazar, S. W., \& Buckner, R. L. (2009). Intrinsic Functional Connectivity As a Tool For Human Connectomics: Theory, Properties, and Optimization. Journal of Neurophysiology, 103(1), 297-321. https://doi.org/10.1152/jn.00783.2009 
Vieira, B. H., Rondinoni, C., \& Garrido Salmon, C. E. (2020). Evidence of regional associations between age-related inter-individual differences in resting-state functional connectivity and cortical thinning revealed through a multi-level analysis. Neurolmage, 211, 116662.

https://doi.org/10.1016/j.neuroimage.2020.116662

Vincent, J. L., Kahn, I., Snyder, A. Z., Raichle, M. E., \& Buckner, R. L. (2008). Evidence for a Frontoparietal Control System Revealed by Intrinsic Functional Connectivity. Journal of Neurophysiology, 100(6), 3328-3342. https://doi.org/10.1152/jn.90355.2008

Vogel, J. W., Vachon-Presseau, E., Pichet Binette, A., Tam, A., Orban, P., La Joie, R., Savard, M., Picard, C., Poirier, J., Bellec, P., Breitner, J. C. S., \& Villeneuve, S. (2018). Brain properties predict proximity to symptom onset in sporadic Alzheimer's disease. Brain, 141(6), 1871-1883. https://doi.org/10.1093/brain/awy093

Voss, M. W., Weng, T. B., Burzynska, A. Z., Wong, C. N., Cooke, G. E., Clark, R., Fanning, J., Awick, E., Gothe, N. P., Olson, E. A., Mcauley, E., \& Kramer, A. F. (2016). Neurolmage Fitness, but not physical activity, is related to functional integrity of brain networks associated with aging. Neurolmage, 131, 113-125. https://doi.org/10.1016/j.neuroimage.2015.10.044

Whitfield-Gabrieli, \& Nieto-Castanon, A. (2012). Conn: A Functional Connectivity Toolbox for Correlated and Anticorrelated Brain Networks. Brain Connectivity, 2(3), 125-141. https://doi.org/10.1089/brain.2012.0073

Yarkoni, T., \& Westfall, J. (2017). Choosing Prediction Over Explanation in Psychology: Lessons From Machine Learning. Perspectives on Psychological Science: $A$ Journal of the Association for Psychological Science, 12(6), 1100-1122. https://doi.org/10.1177/1745691617693393

Yeo, B. T. T., Krienen, F. M., Sepulcre, J., Sabuncu, M. R., Lashkari, D., Hollinshead, M., Roffman, J. L., Smoller, J. W., Zöllei, L., Polimeni, J. R., Fischl, B., Liu, H., \& Buckner, R. L. (2011). The organization of the human cerebral cortex estimated by intrinsic functional connectivity. Journal of Neurophysiology, 106(3), 11251165. https://doi.org/10.1152/jn.00338.2011 
